\title{
Knowledge and Adoption of Climate Resilient Agricultural Technologies by Paddy Growers
}

\author{
K.V. Manjunath ${ }^{1}$, K. Shivaramu ${ }^{2 *}$ and M.A. Murthy ${ }^{3}$ \\ ${ }^{1}$ Department of Agricultural Extension, University of Agricultural Sciences, GKVK, \\ Bengaluru, Karnataka-560 065, India \\ ${ }^{2}$ Information Specialist, Directorate of Extension, University of Agricultural Sciences, Hebbal, \\ Bengaluru, Karnataka-560 024, India \\ ${ }^{3}$ Directorate of Extension, University of Agricultural Sciences, Hebbal, Bengaluru, \\ Karnataka-560 024, India \\ *Corresponding author
}

A B S T R A C T

The present study was conducted during the year 2017-18 in Mandya

\section{Keywords}

Head reach area, Tail end area, Knowledge,

Adoption. Constraints,

Suggestions, Climate

Resilient Agricultural

Technologies

Article Info

Accepted:

12 July 2019

Available Online:

10 August 2019 district of Karnataka state, India. Two villages each in Head reach and Tail end areas of Krishna Raja Sagar dam were randomly selected. In each selected village 25 paddy growers were personally interviewed by using pre-tested interview schedule. Thus, 100 farmers constituted the sample size. The data collected was analyzed using percentages and ' $t$ ' test. The results of the study reveals that, Majority of the Head reach farmers have low-medium, whereas Majority of the Tail end farmers has medium-high knowledge and adoption. Among the constrains faced by Head reach farmers, lack of sufficient knowledge and guidance on the Climate Resilient Agricultural Technologies ranked first. On the other hand untimely release and improper scheduling of water in the canals ranked first among the problems faced by Tail end farmers.

\section{Introduction}

Indian agriculture is highly dependent on monsoon rains and a close link exists between climate and water resources. The effects of change in climate are global, but countries like India are more vulnerable in view of the high population depends on agriculture. In India, significant negative impact have been implied with medium-term (2010-2039) climate change, predicted to reduce yields by $4.5-9 \%$, depending on the magnitude and distribution of warming. Since agriculture makes up roughly $16 \%$ of India's GDP, a $4.5-9 \%$ negative impact on production implies a cost of climate change to be roughly up to $1.5 \%$ of GDP per year (Venkateswarlu et al., 2013). Climate change is a change in the statistical 
dispensation of weather patterns, when that change lasts for an external period of time. This may refer to alteration in average weather conditions or in the variations of weather around long - term average conditions. The productivity of most cereals would decrease due to increase in temperature and $\mathrm{CO}_{2}$ and the decrease in water availability. There will be a projected loss of $10-40 \%$ in crop production by 2100 if no adaptation measures are taken. A degree Celsius increase in temperature may reduce yields of major food crops by 3-7\% (IPCC, 2012). Rice production is slated to decrease by almost a ton/hectare if the temperature goes up by $2^{\circ} \mathrm{C}$. Rice production is and will be affected by changes in climatic factors like irregular rainfall, long dry spells during wet season (damaging young plants), drought and floods all having an effect on yields. This has also caused outbreaks of pests and diseases, with large losses of crops and harvested products.

Number of methods and practices are being adopted to address climate change challenges by altering cropping patterns, planting dates and farm management techniques. Embankments have been built to save paddy fields from the floods and drought and submergence tolerant varieties of rice are being developed and distributed by government and private organizations. In addition, many farmers are diversifying their production systems, growing other cereals, vegetables and rearing fish and animals. The development of advanced modeling techniques, mapping the effect of climate change on rice growing regions and providing crop insurance are other examples of managing risks and reducing vulnerability. With this background the present study was undertaken with following specific objectives;

To assess the Knowledge level of Paddy growers on Climate Resilient Agricultural Technologies
To find out the extent of Adoption of Climate Resilient Agricultural Technologies by Paddy growers; and

To identify the Constraints of Paddy growers for greater Adoption of Climate Resilient Agricultural Technologies

\section{Materials and Methods}

The study was conducted in Mandya district of Karnataka state. Ex-post facto research design was adopted. Two villages each in Tail end area and Head reach area of Krishna Raj Sagar Dam were randomly selected in consultation with Karnataka State Department of Agriculture (KSDA) Officers, Zonal Agricultural Research Station (ZARS) / Krishi Vigyan Kendra (KVK) Scientists. In each selected village 25 Paddy growing farmers were randomly selected. Thus, 100 farmers constituted the sample for the study. The selected respondents were personally interviewed using pre-tested interview schedule. The items on Climate Resilient Agricultural Technologies in the schedule were developed by referring to literature and consulting experts. Finally, 44 items were considered under five major Headings. viz., Agronomic practices, Soil Fertility Management, Water Management, Pest and Disease Management and Others. The data was tabulated and analyzed using percentages and ' $\mathrm{t}$ ' test.

\section{Results and Discussion}

Overall knowledge level of paddy growers on climate resilient agricultural technologies in paddy

In Head reach area, Overall Knowledge level of Paddy growers with respect to Climate Resilient Agricultural Technologies reveals that slightly less than two fifth $(38 \%)$ of the respondents had medium level of knowledge 
followed by the low (36\%) and high level (26 $\%$ ) of knowledge (Table 1). whereas in the Tail end area, two fifth (42\%) of the respondents had medium level of knowledge followed by high (32\%) and low level (26\%) of knowledge.

Knowledge level of paddy growers with respect to specific climate resilient agricultural technologies

The statement-wise knowledge level of paddy growers with respect to Specific Climate Resilient Agricultural Technologies in paddy cultivation is presented in the table 2 .

In both Head reach and Tail end area, majority of the farmers had more knowledge on practices like summer ploughing, field sanitation, improved land levelling practices, puddling at right time, pre germination of paddy seeds, trimming of top of the aged seedlings before transplanting during late planting, maintaining closer spacing of aged seedlings, increasing number of aged seedlings per hill, maintaining thin film of water for suppression of weeds and avoiding standing water under low lying area to prevent salinity and alkalinity.

In both Head reach and Tail end area majority of the farmers had medium level of knowledge on practices like use of rotary weeder for weed management, crop rotation with pulses, soil testing, soil test based fertilizer application, application of organic manures at right time, use of green manure and green leaf manure at right time, application of recommended quantity of fertilizers, raising healthy seedlings and pest and disease tolerant varieties.

In both Head reach and Tail end area, farmers had low knowledge on the practices like use of bio-fertilizers such as azolla and azospirullum, nitrogen application based on leaf colour chart, incorporation of rice straw under mechanical harvesting, fertigation, drip irrigation, sprinkler irrigation and use of pheromone and light traps.

On the contrary Tail end farmers had more knowledge on water saving Climate Resilient Agricultural practices like SRI method, drum seeding, aerobic paddy, direct seeded paddy and alternate wetting and drying when compared to the Head reach farmers.

Test of significance between tail end and head reach paddy growers with respect to knowledge on climate resilient agricultural technologies

Table 3 depicts the mean scores of Knowledge levels of Head reach and Tail end farmers. As it is evident that, the farmers in Head reach area had obtained a relatively lesser mean score of 26.11, while the farmers in Tail end area had a mean score of 31.70 . Further, the tvalue showed significant difference between knowledge level of Head reach and Tail end farmers at 5 per cent level of probability.

Overall adoption of climate resilient agricultural technologies by paddy growers

The results presented in Table 4 reveals that in Head reach area, two fifth $(40 \%)$ of the respondents had low adoption level followed by medium (36\%) and high adoption level (24 $\%)$. Whereas in the Tail end area, two fifth (40 $\%$ ) of the respondents had medium adoption level followed by high (32\%) and low level $(28 \%)$ of adoption.

Adoption of specific climate resilient agricultural technologies by paddy growers

The Adoption of Specific Climate Resilient Agricultural Technologies by Paddy growers is presented in Table 5. In both Head reach and Tail end area majority of the respondents 
have adopted the practices like summer ploughing, puddling at the right time, pregermination of paddy seeds, trimming of top of the aged seedlings before transplanting during late planting, maintaining closer spacing of aged seedlings and increasing number of aged seedling per hill.

In both Head reach and Tail end area only notable number of respondents have partially adopted the technologies like field sanitation, improved land leveling, maintaining thin film of water for suppression of weeds, use of organic manures, green leaf and green manure, applying recommended quantity of fertilizers and application of neem coated urea.

In both Head reach and Tail end area significant number of respondents have not adopted the technologies like; application of pre and post emergent herbicide, seed treatment with salt water, contingency crop planning and crop rotation with pulses, use of bio fertilizers, nitrogen application based on leaf colour chart, rice straw incorporation under mechanical harvesting, fertigation, drip irrigation, sprinkler irrigation, seed treatment with fungicide / bio-agents, growing pest and disease tolerant varieties, use of pheromone and light traps, alternate wetting \& drying, more spacing to control Brown Plant Hopper $(\mathrm{BPH})$, pulling of rope to dislodge paddy caseworm, destruction of rice stubbles and vector host plants to avoid pathogen build up.

Test of significance between tail end and head reach paddy growers with respect to extent of adoption of climate resilient agricultural technologies

Table 6 depicts the mean scores of Adoption levels of Head reach and Tail end farmers. As it is evident that, the farmers in Head reach area had obtained a relatively lesser mean score of 23.99, while the farmers in Tail end area had a mean score of 30.61. Further, the tvalue showed the significant difference between adoption level of Head reach and Tail end farmers at 5 per cent level of probability.

Constraints in adoption of climate resilient agricultural technologies as perceived by paddy growers

The major constraints perceived by Head reach farmers in Adoption of Climate Resilient Agricultural Technologies (Table 7).

Were lack of sufficient knowledge and guidance on the Climate Resilient Agricultural Technologies (Rank I), limited extension activities on Climate Resilient Agricultural Technologies (Rank II), non-availability of labour to adopt climate resilient agricultural technologies (Rank III). On the other hand the major constraints perceived by the Tail end farmers in adoption of Climate Resilient Agricultural Technologies were, untimely release and improper scheduling of water in the canals (Rank I), uneven and untimely rainfall 1 (Rank II), non-availability of labour to adopt Climate Resilient Agricultural Technologies (Rank III).

The Tail end area farmers had medium-high knowledge and adoption level as compared to the Head reach farmers who had low-medium knowledge and adoption level which may be due to the situational factor like acute water shortage and Tail end farmers had more extension contact with the subject matter specialists (SMS's) of Krishi Vignan Kendra $(\mathrm{KVK})$, Mandya and they were participated in extension educational activities like demonstrations, group meetings, field days etc., to a greater extent. Further, the farmers in Head reach area had obtained a relatively lesser mean score, while the farmers in Tail end area had a higher mean score with respect to knowledge and adoption scores. 
Table.1 Overall knowledge level of paddy growers with respect to climate resilient agricultural technologies

\begin{tabular}{|c|c|c|c|c|c|c|}
\hline \multirow[t]{2}{*}{ Knowledge Level } & \multicolumn{2}{|c|}{ Head reach } & \multicolumn{2}{|c|}{ Tail end } & \multicolumn{2}{|c|}{ Total } \\
\hline & No. & $\%$ & No. & $\%$ & No. & $\%$ \\
\hline Low & 18 & 36.00 & 13 & 26.00 & 31 & 31.00 \\
\hline Medium & 19 & 38.00 & 21 & 42.00 & 40 & 40.00 \\
\hline High & 13 & 26.00 & 16 & 32.00 & 29 & 29.00 \\
\hline Total & 50 & 100.00 & $\mathbf{5 0}$ & 100.00 & 100 & 100.00 \\
\hline
\end{tabular}

Table. 2 Knowledge level of paddy growers on specific climate resilient agricultural technologies

\begin{tabular}{|c|c|c|c|c|c|c|c|}
\hline \multirow{3}{*}{$\begin{array}{l}\text { Sl. } \\
\text { No. }\end{array}$} & \multirow{3}{*}{ Technologies } & \multicolumn{6}{|c|}{ Complete knowledge } \\
\hline & & \multicolumn{2}{|c|}{ Head reach } & \multicolumn{2}{|c|}{ Tail end } & \multicolumn{2}{|c|}{ Total } \\
\hline & & No. & $\%$ & No. & $\%$ & No. & $\%$ \\
\hline I & Agronomic practices & & & & & & \\
\hline 1 & Summer ploughing & 44 & 88.00 & 47 & 94.00 & 91 & 91.00 \\
\hline 2 & $\begin{array}{l}\text { Field sanitation, bund trimming, cleaning and proper disposal } \\
\text { of waste }\end{array}$ & 47 & 94.00 & 45 & 90.00 & 92 & 92.00 \\
\hline 3 & Improved Land levelling practices & 49 & 98.00 & 47 & 94.00 & 96 & 96.00 \\
\hline 4 & Puddling at right time to manage weeds and to retain water & 41 & 82.00 & 39 & 78.00 & 80 & 80.00 \\
\hline 5 & Application of pre and post emergent herbicides & 33 & 66.00 & 28 & 56.00 & 61 & 61.00 \\
\hline 6 & Selection of good seeds through salt water treatment & 9 & 18.00 & 14 & 28.00 & 26 & 26.00 \\
\hline 8 & Direct seeded paddy & 6 & 12.00 & 16 & 32.00 & 22 & 22.00 \\
\hline 9 & Drum seeded of paddy & 22 & 44.00 & 38 & 76.00 & 60 & 60.00 \\
\hline 10 & System of Rice Intensification (SRI) method of paddy & 31 & 62.00 & 40 & 80.00 & 71 & 71.00 \\
\hline 11 & Aerobic paddy & 17 & 34.00 & 26 & 52.00 & 43 & 43.00 \\
\hline 12 & $\begin{array}{l}\text { Trimming of top of the aged Seedlings before transplanting } \\
\text { during late planting }\end{array}$ & 40 & 80.00 & 41 & 82.00 & 81 & 81.00 \\
\hline 13 & Maintaining closer spacing of aged seedlings & 42 & 84.00 & 40 & 80.00 & 82 & 82.00 \\
\hline 14 & Increasing number of aged seedlings per hill & 44 & 88.00 & 43 & 86.00 & 87 & 87.00 \\
\hline 15 & Use of rotary weeder for weed management & 30 & 60.00 & 36 & 72.00 & 56 & 56.00 \\
\hline 16 & Maintaining thin film of water for suppression of weeds & 42 & 84.00 & 39 & 78.00 & 81 & 81.00 \\
\hline 17 & Contingency crop planning & 22 & 44.00 & 31 & 62.00 & 53 & 53.00 \\
\hline 18 & Crop rotation with pulses & 29 & 58.00 & 36 & 72.00 & 65 & 65.00 \\
\hline II & Soil Fertility Management & & & & & & \\
\hline 19 & Soil testing, soil test based fertilizer application & 31 & 62.00 & 34 & 68.00 & 65 & 65.00 \\
\hline 20 & Use of biofertilizers & & & & & & \\
\hline i) & Use of Azolla & 11 & 22.00 & 18 & 36.00 & 29 & 29.00 \\
\hline ii) & Use of Azospirillum & 2 & 4.00 & 9 & 18.00 & 11 & 11.00 \\
\hline 21 & Application of organic manures at right time & 33 & 66.00 & 31 & 62.00 & 64 & 64.00 \\
\hline 22 & Use of green manure and green leaf manure at right time & 32 & 64.00 & 30 & 60.00 & 62 & 62.00 \\
\hline 23 & Application of recommended quantity of fertilizers & 27 & 54.00 & 30 & 60.00 & 57 & 57.00 \\
\hline 24 & Application of neem coated urea & 45 & 90.00 & 46 & 92.00 & 91 & 91.00 \\
\hline 25 & Nitrogen application based on leaf colour chart & 2 & 4.00 & 1 & 2.00 & 3 & 3.00 \\
\hline
\end{tabular}




\begin{tabular}{|c|c|c|c|c|c|c|c|}
\hline 26 & Incorporation of rice straw under mechanical harvesting & 3 & 6.00 & 2 & 4.00 & 5 & 5.00 \\
\hline 27 & Fertigation & 2 & 4.00 & 3 & 6.00 & 5 & 5.00 \\
\hline \multicolumn{8}{|c|}{ III Water Management } \\
\hline 28 & Draining out of excess Water (aerobic \& SRI) & 16 & 32.00 & 25 & 50.00 & 41 & 41.00 \\
\hline 29 & $\begin{array}{l}\text { Avoiding standing water under low lying area to prevent } \\
\text { salinity and alkalinity }\end{array}$ & 42 & 84.00 & 41 & 82.00 & 83 & 83.00 \\
\hline 30 & Alternate wetting and drying & 21 & 42.00 & 30 & 60.00 & 51 & 51.00 \\
\hline 31 & Drip irrigation & 8 & 16.00 & 18 & 36.00 & 26 & 26.00 \\
\hline 32 & Sprinkler irrigation & 3 & 6.00 & 6 & 12.00 & 9 & 9.00 \\
\hline IV & \multicolumn{2}{|l|}{ Pest and Disease Management } & & & & & \\
\hline 33 & Raising healthy seedlings & 35 & 70.00 & 38 & 76.00 & 73 & 73.00 \\
\hline 34 & Seed treatment with Fungicide / bio-agents & 15 & 30.00 & 19 & 38.00 & 34 & 34.00 \\
\hline 35 & Growing Pest and disease tolerant varieties & 24 & 48.00 & 25 & 50.00 & 49 & 49.00 \\
\hline 36 & Use of pheromone traps to control stem borer & 2 & 4.00 & 1 & 2.00 & 3 & 3.00 \\
\hline 37 & Alternate wetting \& Drying \& more spacing to control BPH & 17 & 34.00 & 21 & 42.00 & 38 & 38.00 \\
\hline 38 & Pulling of rope to dislodge paddy caseworm & 9 & 18.00 & 11 & 22.00 & 20 & 20.00 \\
\hline 39 & Use of light traps for nocturnal, sucking pests & 5 & 10.00 & 7 & 14.00 & 12 & 12.00 \\
\hline 40 & Clipping of rice seedlings for management of rice stem borer & 13 & 26.00 & 12 & 24.00 & 25 & 25.00 \\
\hline 41 & $\begin{array}{l}\text { Destruction of rice stubbles and vector host plants to avoid } \\
\text { pathogen build up }\end{array}$ & 17 & 34.00 & 19 & 38.00 & 36 & 36.00 \\
\hline $\mathbf{V}$ & Others & & & & & & \\
\hline 42 & Growing drought tolerant varieties & 18 & 36.00 & 29 & 58.00 & 47 & 47.00 \\
\hline 43 & Growing saline soil tolerant varieties & 3 & 6.00 & 1 & 2.00 & 4 & 4.00 \\
\hline 44 & $\begin{array}{l}\text { Growing recommended Varieties suitable for different sowing } \\
\text { period }\end{array}$ & 47 & 92.00 & 45 & 90.00 & 92 & 92.00 \\
\hline
\end{tabular}

Table.3 Test of significance between tail end and head reach paddy growers with respect to knowledge on climate resilient agricultural technologies

$(\mathrm{N}=100)$

\begin{tabular}{|c|c|c|}
\hline \multirow{2}{*}{ Paddy growers } & \multicolumn{2}{|c|}{ Knowledge level } \\
\cline { 2 - 3 } & Mean Score & 't' test \\
\hline Head reach & 26.11 & $1.8203^{*}$ \\
\hline Tail end & 31.70 & \\
\hline
\end{tabular}

*Significant at $5 \%$ level

Table.4 Overall adoption of climate resilient agricultural technologies by paddy growers

\begin{tabular}{|c|c|c|c|c|c|c|}
\hline \multirow{3}{*}{$\begin{array}{c}\text { Adoption } \\
\text { Level }\end{array}$} & \multirow{2}{*}{\multicolumn{2}{|c|}{ Head reach }} & \multirow{2}{*}{\multicolumn{2}{|c|}{ Tail end }} & \multirow{2}{*}{\multicolumn{2}{|c|}{ Total $(\mathrm{N}=100)$}} \\
\hline & & & & & & \\
\hline & No. & $\%$ & No. & $\%$ & No. & $\%$ \\
\hline Low & 20 & 40.00 & 14 & 28.00 & 34 & 34.00 \\
\hline Medium & 18 & 36.00 & 20 & 40.00 & 38 & 38.00 \\
\hline High & 12 & 24.00 & 16 & 32.00 & 28 & 28.00 \\
\hline Total & 50 & 100.00 & 50 & 100.00 & 100 & 100.00 \\
\hline
\end{tabular}


Table.5 Adoption of specific climate resilient technologies by paddy growers

$(\mathrm{N}=100)$

\begin{tabular}{|c|c|c|c|c|c|c|c|c|c|c|c|c|c|c|c|c|c|c|c|}
\hline \multirow{3}{*}{$\begin{array}{l}\text { Sl. } \\
\text { No. }\end{array}$} & \multirow[t]{3}{*}{ Technologies } & \multicolumn{6}{|c|}{ Full adoption } & \multicolumn{6}{|c|}{ Partial adoption } & \multicolumn{6}{|c|}{ Non adoption } \\
\hline & & \multicolumn{2}{|c|}{ Head reach } & \multicolumn{2}{|c|}{ Tail end } & \multicolumn{2}{|c|}{ Total } & \multicolumn{2}{|c|}{ Head reach } & \multicolumn{2}{|c|}{ Tail end } & \multicolumn{2}{|c|}{ Total } & \multicolumn{2}{|c|}{ Head reach } & \multicolumn{2}{|c|}{ Tail end } & \multicolumn{2}{|c|}{ Total } \\
\hline & & No. & $\%$ & No. & $\%$ & No. & $\%$ & No. & $\%$ & No. & $\%$ & No. & $\%$ & No. & $\%$ & No. & $\%$ & No. & $\%$ \\
\hline I & Agronomic Practices & & & & & & & & & & & & & & & & & & \\
\hline 1 & Summer ploughing & 42 & 84.00 & 45 & 90.00 & 87 & 87.00 & 00 & 00.00 & 00 & 00.00 & 00 & 00.00 & 8 & 16.00 & 5 & 10.00 & 13 & 13.00 \\
\hline 2 & $\begin{array}{l}\text { Field sanitation; bund trimming, cleaning and } \\
\text { proper disposal of waste }\end{array}$ & 30 & 60.00 & 28 & 56.00 & 58 & 58.00 & 20 & 40.00 & 22 & 44.00 & 42 & 42.00 & 00 & 00.00 & 00 & 00.00 & 00 & 00.00 \\
\hline 3 & Improved land leveling practices & 23 & 46.00 & 22 & 44.00 & 45 & 45.00 & 27 & 54.00 & 28 & 56.00 & 55 & 55.00 & 00 & 00.00 & 00 & 00.00 & 00 & 00.00 \\
\hline 4 & $\begin{array}{l}\text { Puddling at the right time to manage weeds } \\
\text { and to retain water }\end{array}$ & 29 & 58.00 & 30 & 60.00 & 59 & 59.00 & 21 & 42.00 & 20 & 40.00 & 41 & 41.00 & 00 & 00.00 & 00 & 00.00 & 00 & 00.00 \\
\hline 5 & $\begin{array}{l}\text { Application of pre and post emergent } \\
\text { herbicides }\end{array}$ & 21 & 42.00 & 19 & 38.00 & 40 & 40.00 & 00 & 00.00 & 00 & 00.00 & 00 & 00.00 & 29 & 58.00 & 31 & 62.00 & 60 & 60.00 \\
\hline 6 & $\begin{array}{l}\text { Selection of good seeds through salt water } \\
\text { treatment }\end{array}$ & 2 & 4.00 & 3 & 6.00 & 5 & 5.00 & 00 & 00.00 & 00 & 00.00 & 00 & 00.00 & 48 & 96.00 & 47 & 94.00 & 95 & 95.00 \\
\hline 7 & Pregermination of paddy seeds & 47 & 94.00 & 48 & 96.00 & 95 & 95.00 & 00 & 00.00 & 00 & 00.00 & 00 & 00.00 & 3 & 6.00 & 2 & 4.00 & 5 & 5.00 \\
\hline 8 & Direct seeded paddy & 3 & 6.00 & 9 & 18.00 & 12 & 12.00 & 00 & 00.00 & 00 & 00.00 & 00 & 00.00 & 47 & 94.00 & 41 & 82.00 & 88 & 88.00 \\
\hline 9 & Drum seeded paddy & 9 & 18.00 & 26 & 52.00 & 35 & 35.00 & 00 & 00.00 & 00 & 00.00 & 00 & 00.00 & 41 & 82.00 & 24 & 48.00 & 65 & 65.00 \\
\hline 10 & $\begin{array}{l}\text { System of Rice intensification (SRI)method } \\
\text { of paddy }\end{array}$ & 18 & 36.00 & 30 & 60.00 & 48 & 48.00 & 00 & 00.00 & 00 & 00.00 & 00 & 00.00 & 32 & 64.00 & 20 & 40.00 & 52 & 52.00 \\
\hline 11 & Aerobic paddy & 4 & 8.00 & 18 & 36.00 & 22 & 22.00 & 00 & 00.00 & 00 & 00.00 & 00 & 00.00 & 46 & 92.00 & 32 & 64.00 & 78 & 78.00 \\
\hline 12 & $\begin{array}{l}\text { Trimming of top of the aged seedlings before } \\
\text { transplanting during late planting }\end{array}$ & 30 & 60.00 & 33 & 66.00 & 63 & 63.00 & 00 & 00.00 & 00 & 00.00 & 00 & 00.00 & 20 & 40.00 & 17 & 34.00 & 37 & 37.00 \\
\hline 13 & Maintaining closer spacing of aged seedlings & 28 & 56.00 & 31 & 62.00 & 59 & 59.00 & 00 & 00.00 & 00 & 00.00 & 00 & 00.00 & 22 & 44.00 & 19 & 38.00 & 41 & 41.00 \\
\hline 14 & Increasing number of aged seedlings per hill & 31 & 62.00 & 30 & 60.00 & 61 & 61.00 & 00 & 00.00 & 00 & 00.00 & 00 & 00.00 & 19 & 38.00 & 20 & 40.00 & 39 & 39.00 \\
\hline 15 & Use of rotary weeder for weed management & 11 & 22.00 & 13 & 26.00 & 24 & 24.00 & 00 & 00.00 & 00 & 00.00 & 00 & 00.00 & 39 & 78.00 & 37 & 74.00 & 76 & 76.00 \\
\hline 16 & $\begin{array}{l}\text { Maintaining thin film of water for } \\
\text { suppression of weeds }\end{array}$ & 17 & 34.00 & 14 & 28.00 & 31 & 31.00 & 33 & 66.00 & 36 & 72.00 & 69 & 69.00 & 00 & 00.00 & 00 & 00.00 & 00 & 00.00 \\
\hline 17 & Contingency crop planning & 14 & 28.00 & 18 & 36.00 & 32 & 32.00 & 00 & 00.00 & 00 & 00.00 & 00 & 00.00 & 36 & 72.00 & 32 & 64.00 & 68 & 68.00 \\
\hline 18 & Crop rotation with pulses & 16 & 32.00 & 26 & 52.00 & 42 & 42.00 & 00 & 00.00 & 00 & 00.00 & 00 & 00.00 & 34 & 68.00 & 24 & 48.00 & 58 & 58.00 \\
\hline II & Soil Fertility Management & & & & & & & & & & & & & & & & & & \\
\hline 19 & $\begin{array}{l}\text { Soil testing, soil test based fertilizer } \\
\text { application }\end{array}$ & 14 & 28.00 & 20 & 40.00 & 34 & 34.00 & 00 & 00.00 & 00 & 00.00 & 00 & 00.00 & 36 & 72.00 & 30 & 60.00 & 66 & 66.00 \\
\hline 20 & Use of Biofertilizers & & & & & & & & & & & & & & & & & & \\
\hline i) & use of azolla & 5 & 10.00 & 2 & 4.00 & 7 & 7.00 & 00 & 00.00 & 00 & 00.00 & 00 & 00.00 & 45 & 90.00 & 48 & 96.00 & 93 & 93.00 \\
\hline ii) & use of azospirillum & 2 & 4.00 & 1 & 2.00 & 3 & 3.00 & 0 & 00.00 & 0 & 00.00 & 00 & 00.00 & 48 & 96.00 & 49 & 98.00 & 97 & 97.00 \\
\hline 21 & Application of organic manures at right time & 6 & 12.00 & 9 & 18.00 & 15 & 15.00 & 43 & 86.00 & 39 & 78.00 & 82 & 82.00 & 1 & 2.00 & 2 & 4.00 & 3 & 3.00 \\
\hline
\end{tabular}


Int.J.Curr.Microbiol.App.Sci (2019) 8(8): 1430-1442

\begin{tabular}{|c|c|c|c|c|c|c|c|c|c|c|c|c|c|c|c|c|c|c|c|}
\hline 22 & Use of green manure and green leaf manure & 7 & 14.00 & 6 & 12.00 & 13 & 13.00 & 30 & 60.00 & 27 & 54.00 & 57 & 57.00 & 13 & 26.00 & 17 & 34.00 & 30 & 30.00 \\
\hline 23 & $\begin{array}{l}\text { Application of recommended quantity of } \\
\text { fertilizers }\end{array}$ & 8 & 16.00 & 9 & 18.00 & 17 & 17.00 & 42 & 84.00 & 41 & 82.00 & 83 & 83.00 & 00 & 00.00 & 00 & 00.00 & 00 & 00.00 \\
\hline 24 & Application of neem coated urea & 11 & 22.00 & 19 & 38.00 & 30 & 30.00 & 39 & 78.00 & 31 & 62.00 & 70 & 70.00 & 00 & 00.00 & 00 & 00.00 & 00 & 00.00 \\
\hline 25 & $\begin{array}{l}\text { Nitrogen application based on leaf colour } \\
\text { chart }\end{array}$ & 00 & 00.00 & 00 & 00.00 & 00 & 00.00 & 00 & 00.00 & 00 & 00.00 & 00 & 00.00 & 100 & 100.00 & 100 & 100.0 & 100 & 100.00 \\
\hline 26 & $\begin{array}{l}\text { Rice straw incorporation under mechanical } \\
\text { harvesting }\end{array}$ & 00 & 00.00 & 00 & 00.00 & 00 & 00.00 & 00 & 00.00 & 00 & 00.00 & 00 & 00.00 & 50 & 100.00 & 50 & 100.00 & 100 & 100.00 \\
\hline 27 & Fertigation & 00 & 00.00 & 00 & 00.00 & 00 & 00.00 & 00 & 00.00 & 00 & 00.00 & 00 & 00.00 & 50 & 100.00 & 50 & 100.00 & 100 & 100.00 \\
\hline III & Water Management & & & & & & & & & & & & & & & & & & \\
\hline 28 & Draining out excess water (aerobic \& SRI) & 11 & 22.00 & 18 & 36.00 & 29 & 29.00 & 00 & 00.00 & 00 & 00.00 & 00 & 00.00 & 39 & 78.00 & 32 & 64.00 & 71 & 71.00 \\
\hline 29 & $\begin{array}{l}\text { Avoiding standing water under low lying } \\
\text { area to prevent salinity and alkalinity }\end{array}$ & 9 & 18.00 & 00 & 00.00 & 9 & 9.00 & 00 & 00.00 & 00 & 00.00 & 00 & 00.00 & 41 & 82.00 & 50 & 100.00 & 91 & 91.00 \\
\hline 30 & Alternate wetting and drying & 21 & 42.00 & 26 & 52.00 & 47 & 47.00 & 00 & 00.00 & 00 & 00.00 & 00 & 00.00 & 29 & 58.00 & 24 & 48.00 & 53 & 53.00 \\
\hline 31 & Drip irrigation & 00 & 00.00 & 00 & 00.00 & 00 & 00.00 & 00 & 00.00 & 00 & 00.00 & 00 & 00.00 & 50 & 100.00 & 50 & 100.00 & 100 & 100.00 \\
\hline 32 & Sprinkler irrigation & 00 & 00.00 & 00 & 00.00 & 00 & 00.00 & 00 & 00.00 & 00 & 00.00 & 00 & 00.00 & 50 & 100.00 & 50 & 100.00 & 100 & 100.00 \\
\hline IV & Pest and Disease Management & & & & & & & & & & & & & & & & & & \\
\hline 33 & Raising healthy seedlings & 18 & 36.00 & 19 & 38.00 & 37 & 37.00 & 32 & 64.00 & 31 & 62.00 & 63 & 63.00 & 00 & 00.00 & 00 & 00.00 & 00 & 00.00 \\
\hline 34 & $\begin{array}{l}\text { Seed treatment with fungicide/bioagents } \\
\text { (Trichoderma, Azosprillum) }\end{array}$ & 10 & 20.00 & 12 & 24.00 & 22 & 22.00 & 00 & 00.00 & 00 & 00.00 & 00 & 00.00 & 40 & 80.00 & 38 & 76.00 & 78 & 78.00 \\
\hline 35 & Growing Pest and disease tolerant varieties & 11 & 22.00 & 14 & 28.00 & 25 & 25.00 & 00 & 00.00 & 00 & 00.00 & 00 & 00.00 & 39 & 78.00 & 36 & 72.00 & 75 & 75.00 \\
\hline 36 & $\begin{array}{l}\text { Use of pheromone traps to control stem borer } \\
\text { attack }\end{array}$ & 00 & 00.00 & 00 & 00.00 & 00 & 00.00 & 00 & 00.00 & 00 & 00.00 & 00 & 00.00 & 50 & 100.00 & 50 & 100.00 & 100 & 100.00 \\
\hline 37 & $\begin{array}{l}\text { Alternate wetting \& drying, more spacing to } \\
\text { control BPH }\end{array}$ & 6 & 12.00 & 8 & 16.00 & 14 & 14.00 & 00 & 00.00 & 00 & 00.00 & 00 & 00.00 & 44 & 88.00 & 42 & 84.00 & 86 & 86.00 \\
\hline 38 & Pulling of rope to dislodge paddy caseworm & 1 & 2.00 & 2 & 4.00 & 3 & 3.00 & 00 & 00.00 & 00 & 00.00 & 00 & 00.00 & 49 & 98.00 & 48 & 96.00 & 97 & 97.00 \\
\hline 39 & $\begin{array}{l}\text { Use of light traps for nocturnal and sucking } \\
\text { pests }\end{array}$ & 2 & 4.00 & 1 & 2.00 & 3 & 3.00 & 00 & 00.00 & 00 & 00.00 & 00 & 00.00 & 48 & 96.00 & 49 & 98.00 & 97 & 97.00 \\
\hline 40 & $\begin{array}{l}\text { Clipping of rice seedlings for management of } \\
\text { rice stem borer }\end{array}$ & 9 & 18.00 & 8 & 16.00 & 17 & 17.00 & 00 & 00.00 & 00 & 00.00 & 00 & 00.00 & 41 & 82.00 & 42 & 84.00 & 83 & 83.00 \\
\hline 41 & $\begin{array}{l}\text { Destruction of rice stubbles and vector host } \\
\text { plants to avoid pathogen build up }\end{array}$ & 3 & 6.00 & 4 & 8.00 & 7 & 7.00 & 00 & 00.00 & 00 & 00.00 & 00 & 00.00 & 47 & $94 . .00$ & 46 & 92.00 & 93 & 93.00 \\
\hline $\mathbf{V}$ & Others & & & & & & & & & & & & & & & & & & \\
\hline 42 & Growing drought tolerant varieties & 4 & 8.00 & 19 & 38.00 & 23 & 23.00 & 00 & 00.00 & 00 & 00.00 & 00 & 00.00 & 46 & 92.00 & 31 & 62.00 & 77 & 77.00 \\
\hline 43 & Growing saline soil tolerant varieties & 2 & 4.00 & 00 & 00.00 & 2 & 2.00 & 00 & 00.00 & 00 & 00.00 & 00 & 00.00 & 48 & 96.00 & 50 & 100.00 & 98 & 98.00 \\
\hline 44 & $\begin{array}{l}\text { Growing recommended varieties suitable for } \\
\text { different sowing period }\end{array}$ & 18 & 36.00 & 17 & 34.00 & 35 & 35.00 & 00 & 00.00 & 00 & 00.00 & 00 & 00.00 & 32 & 64.00 & 33 & 66.00 & 65 & 65.00 \\
\hline
\end{tabular}


Table.6 Test of significance between tail end and head reach paddy growers with respect to extent of adoption of climate resilient agricultural technologies

\begin{tabular}{|c|c|c|}
\hline \multirow{2}{*}{ Paddy growers } & \multicolumn{2}{|c|}{ Extent of adoption } \\
\cline { 2 - 3 } & Mean score & “t” value \\
\hline Head reach & 23.99 & $1.8692 *$ \\
\hline Tail end & 30.61 & \\
\hline
\end{tabular}

* Significant at $5 \%$ level

Table.7 Constraints faced by paddy growers in adoption of climate resilient agricultural technologies

\begin{tabular}{|c|c|c|c|c|}
\hline & \multirow[b]{2}{*}{ Constraints } & & \multicolumn{2}{|c|}{$(\mathrm{N}=100)$} \\
\hline & & Score & $\begin{array}{l}\text { Mean } \\
\text { score }\end{array}$ & Rank \\
\hline $\mathbf{A}$ & Head reach & & & \\
\hline 1 & $\begin{array}{l}\text { Lack of sufficient knowledge and guidance on the Climate } \\
\text { Resilient Agricultural Technologies }\end{array}$ & 133 & 2.66 & I \\
\hline 2 & $\begin{array}{l}\text { Limited extension activities on Climate Resilient Agricultural } \\
\text { Technologies }\end{array}$ & 127 & 2.54 & II \\
\hline 3 & $\begin{array}{l}\text { Non availability of labour to adopt Agricultural Technologies } \\
\text { climate resilient }\end{array}$ & 118 & 2.36 & III \\
\hline 4 & Non availability of critical inputs & 111 & 2.22 & IV \\
\hline 5 & High cost of inputs & 106 & 2.12 & V \\
\hline 6 & Uneven and untimely rainfall & 102 & 2.04 & VI \\
\hline 7 & Power shortage & 90 & 1.80 & VII \\
\hline 8 & Untimely release and improper scheduling of water in the canals & 87 & 1.74 & VIII \\
\hline 9 & Small sized land holdings & 81 & 1.62 & IX \\
\hline 10 & Mono cropping & 72 & 1.44 & $\mathrm{X}$ \\
\hline B & Tail end & & & \\
\hline 1 & Untimely release and improper scheduling of water in the canals & 136 & 2.72 & I \\
\hline 2 & Uneven and untimely rainfall & 130 & 2.60 & II \\
\hline 3 & $\begin{array}{l}\text { Non availability of labour to adopt climate Resilient agricultural } \\
\text { technologies }\end{array}$ & 122 & 2.44 & III \\
\hline 4 & Mono cropping & 121 & 2.42 & IV \\
\hline 5 & $\begin{array}{l}\text { Lack of sufficient knowledge and guidance on the climate } \\
\text { resilient agricultural technologies }\end{array}$ & 114 & 2.28 & V \\
\hline 6 & Power shortage & 108 & 2.16 & VI \\
\hline 7 & Non availability of critical inputs & 108 & 2.16 & VI \\
\hline 8 & $\begin{array}{l}\text { Limited extension activities on climate resilient agricultural } \\
\text { technologies }\end{array}$ & 105 & 2.10 & VII \\
\hline 9 & High cost of inputs & 92 & 1.84 & VIII \\
\hline 10 & Small sized land holdings & 83 & 1.66 & IX \\
\hline
\end{tabular}


The t-value showed significant difference between knowledge and adoption level of Head reach and Tail end farmers at 5 per cent level of probability. Similarly Balakrishnan and Vasanthakumar (2010) revealed that more than half of respondents had medium level of knowledge followed by high level of knowledge and low knowledge level about SRI technology. Also, Thiyagarajan (2011) revealed that majority of the respondents had medium level of knowledge followed by low level and high level of knowledge on SRI cultivation. Further, Jasna (2015) reported that in Karnataka and Jharkhand NICRA farmers had a higher resilient index score than the non- NICRA farmers.

In both Head reach and Tail end area, majority of the farmers had more knowledge and full adoption on practices like summer ploughing, puddling at right time, pre germination of paddy seeds, trimming of top of the aged seedlings before transplanting during late planting, maintaining closer spacing of aged seedlings and increasing number of aged seedlings per hill as these are the low cost and simple technologies which involve less investment and also these are the age old practices which are being followed even from many years. In both Head reach and Tail end area only notable number of respondents has partially adopted the technologies like use of organic manures, green leaf and green manure and applying recommended quantity of fertilizers as fertilizers and manures require cash to purchase and again respondents had partial knowledge. In both Head reach and Tail end area farmers had low knowledge on the practices like use of bio-fertilizers such as azolla \& azospirullum, nitrogen application based on leaf colour chart, incorporation of rice straw under mechanical harvesting, fertigation, drip irrigation, sprinkler irrigation, use of pheromone traps and light traps as these are new to them, complex, difficult to understand and involves more skill. On the contrary Tail end farmers had more knowledge and adoption of water saving Climate Resilient Agricultural practices like SRI method, drum seeding, aerobic paddy, direct seeded paddy and alternate wetting and drying when compared to the Head reach farmers may be due to water shortage, nonrelease and untimely release of canal water, more extension contact and demonstrations and training programmes conducted by the KVK, Mandya. Thiyagarajan (2011) revealed that nearly half of the respondents had high level of adoption in the cultivation of paddy under SRI method followed by medium and low levels of adoption. Friedrich et al., (2012) reported that adoption of Conservation Agriculture has been low (4.72 M ha) in Asia, particularly in South Asia where knowledge and adoption of Conservation Agriculture is on the increase. Pathak et al., (2012) identified ten adaptation options having the highest priority in mitigating climatic vulnerability following experts ranking. These options were climate-ready crop varieties, water-saving technologies, changing planting dates, integrated farming system, growing different crops, integrated pest management, crop insurance, conservation agriculture, improved weather-based agro-advisory and improved nutrient management. Shashidhara (2012) revealed that majority of respondents was in medium level adoption of eco-friendly technologies with respect to adoption on integrated nutrient management, majority of the respondents were not practicing, application of organic manures, selection of crops and cropping pattern, mixed cropping, inter-cultivation practices, application of biofertilizers to soil and use of limited in organic fertilizers in cotton. Evans et al., (2015) revealed that carbon farming programs aim to combat climate change by encouraging land managers to adopt „carbon farming practices $^{e e}$. These practices may involve either sequestering carbon in soils/vegetation, or 
reducing emissions. To sequester carbon in vegetation, land managers can plant trees, protect remnant vegetation, restore native vegetation or reforest degraded lands.

The major constraints perceived by Head reach farmers in Adoption of Climate Resilient Agricultural Technologies were lack of sufficient knowledge and guidance on the Climate Resilient Agricultural Technologies (Rank I), limited extension activities on Climate Resilient Agricultural Technologies (Rank II), non-availability of labour to adopt climate resilient agricultural technologies (Rank III). On the other hand the major constraints perceived by the Tail end farmers in adoption of Climate Resilient Agricultural Technologies were, untimely release and improper scheduling of water in the canals (Rank I), uneven and untimely rainfall 1 (Rank II), non-availability of labour to adopt Climate Resilient Agricultural Technologies (Rank III). Nzeadibe et al., (2011) found that the major constraints in adapting to climate change by farmers in the Niger Delta included lack of information, low awareness level, irregularities of extension services, poor government attention to climate problems, inability to access available information, lack of access to improved crop varieties. However, other constraining factors were ineffectiveness of indigenous methods, no subsidies on planting materials, limited knowledge on adaptation measures, low institutional capacity and absence of government policy on climate change. Shivaramu, et al., (2011) reported that cent percent of drum seedling farmers opinioned that farmers can use drum seeder, but majority of them discontinued the use of drum seeder. The major reasons expressed by them for discontinuance are non-adoption of timely drum seeding practices lead to more problems, maintenance of water in initial stages of crop establishment, birds menace, more price of weedicide and failure in control of Echinoclova and sedges by weedicides. Farmers got additional income of Rs 9363 per hectare by adopting drum seeder technology compared to transplanting through savings. Ahmed (2013) inferred that most of the farmers were well informed about the new development in agriculture and they were ready to adopt the new farming technology but were not in a position to adopt the improved technology at full scale due to certain constraints faced by them in day-today life. The constraints mentioned by farmers were Impurity of seeds, Inadequate irrigation facilities, Erratic supply of electricity, Lack of information about new technology, Lesser contact with extension agencies, Problems of soil testing, water testing, soil salinity etc and Environmental problem. Negash (2013) reported that the constraints to adapt to climate change practices faced by the farmers were lack of information/ knowledge (34.55\%), shortage of money $(23.95 \%)$, and shortage of land (20.40\%), unsuitability of land and poor potential for irrigation (11.50\%), shortage of labour $(5.60 \%)$ and others $(4.00 \%)$ in North Shoa Zone of Amhara region, Ethiopia. Philip et al., (2013) reported that majority of household perceived lack of financial resources as a serious barriers to climate adaptation followed by lack of information on climate change characteristics, lack of institutional capacity to facilitate agricultural adaptation, social barriers and lack of infrastructural development in Sub-Saharan Africa.

The study revealed that, significant number of the respondents in both Head reach area and Tail end area had medium level of knowledge on climate resilient technologies in paddy cultivation therefore it is a matter of concern that still large portion of farmers don't have knowledge on these technologies especially Head reach farmers. Hence there is an immediate necessary to awaken the farming 
community towards Climate Resilient Agricultural technologies which helps to adapt to climate change and increase the production on a sustainable basis.

The study also revealed that major portion of the farmers in Head reach area had low adoption level followed by medium adoption level in the Tail end area. Among them a negligible proportion of farmers have adopted micro irrigation, use of light and pheromone traps, leaf colour chart, seed treatment with salt water, use of bio fertilizers etc., Hence farmers need to be educated, convinced about the importance of Climate Resilient Agricultural technologies. In the long run there is a need to provide required facilities by the State department of Agriculture, besides providing more technical guidance through conducting demonstration in every village and follow up approach.

The major constraints expressed by the Head reach farmers were lack of sufficient knowledge and guidance about the climate resilient technologies, limited extension activities about Climate Resilient agricultural technologies, and non-availability of labour to adopt climate resilient technologies. Hence, the appropriate steps need to be taken by the extension workers and other developmental departments to intensify educational efforts on the adaptation strategies of these climate resilient technologies through extensive use of multimedia, conducting demonstrations and training programs that would create awareness, promote and enable them to adopt climate resilient technologies at a faster rate.

The major constraints perceived by the Tail end farmers are untimely release and improper scheduling of water in the canals, uneven and untimely rainfall, non-availability of labour to adopt climate resilient technologies hence the irrigation department and other concerned line departments officials should involve the local farmers while making decision on irrigation schedule timings and quantity of water release to the canals and also since there is more labour problem extension officials should promote labour saving climate resilient technologies like drum seeding, aerobic paddy etc... and there is a need to develop agricultural equipments suitable to small and marginal farmers.

\section{Acknowledgement}

The research paper is a part of M.Sc (Agri.) Thesis submitted to the University of Agricultural Sciences, Bangalore, Karnataka, India by the first author under the guidance of Second Author.

\section{References}

Ahmed, S., (2013). Factors and Constraints for adopting new Agricultural Technology in Assam with special reference to Nalbari district: An empirical study. Accessed on October 30, 2015 from http://jocipe.Com /jocipe1.html.

Balakrishnan, T. and Vasanthakumar, J., 2010. Knowledge level of System of Rice Intensification (SRI). Technology among farmers in Cuddalore district of Tamil Nadu. International Journal of Current Research. 9: 65-68.

Evans, M.C., Carwardine, J., Fensham, R.J., Butler, D.W., Wilson, K.A., Possingham, H.P., Martin, T. G., 2015. Carbon farming via assisted natural regeneration as a cost effective mechanism for restoring Biodiversity in Agricultural landscapes. Environ. Sci. Policy 50, 114-129.

Friedrich, T., Derpschr and Kassam, A., 2012. Overview of the Global Spread of Conservation Agriculture, field actions science.

Reports 
http://factsreports.revues.org, 194.

IPCC (2012) Managing the Risks of extreme events and disasters to advance Climate change Adaptation summary for policymakers, special report of Intergovernmental panel on Climate change. Accessed on 14th March 2015, from https://www.ipcc.ch/pdf/specialreports/srex/SREX_FD_SPM_final.pdf.

Jasna, V.K., 2015. Impact of Climate Resilient Technologies in Rainfed Agroeco system. M.Sc. Agri Thesis (unpublished). IARI, New Delhi.

Negash Mulatu Debalke., 2013. Determination of farmers Preference for Adaptation strategies to Climate change: Evidence from North Shoa Zone of Amhara region, Ethiopia. http://mpra.ub.uni-muenchen.de/48753.

Nzeadibe, T.C., Egbule, C.L., Chukwuone, N.A. and Agu, V.C., 2011. Climate change Awareness and Adaptation in the Niger Delta Region of Nigeria. African Technology Policy Studies Network (ATPS) Working Paper Series. Nairobi: ATPS.

Pathak, H., Aggarwal, P.K. and Singh, S.D., 2012. Climate Change Impact, Adaptation and Mitigation in Agriculture: Methodology for
Assessment and Applications. Indian Agricultural Research Institute, New Delhi.

Shashidhara, K. K., 2012. Adoption of Ecofriendly Technologies by Cotton growers. Indian Research Journal of extension education, special issue 1: 217-22.

Shivaramu, K., Siddu, H. C. and Ranganatha, A. D., 2011. Economics and Adoption of Drum Seeding Technology by Paddy Growers. Environment \& Ecology, 29(1A): 287-289.

Thiyagarajan, M., 2011. Impact analysis of System of Rice Intensification (SRI) among the Paddy farmers of Coimbatore District. M.Sc. (Agri.) Thesis (Unpublished). Tamil Nadu Agricultural University, Coimbatore, India.

Venkateswarlu, B., Maheswari, M., Srinivasa Rao, M., Rao, V.U.M., Srinivasa Rao, C.H., Reddy, K.S., Ramana, D.B.V., Rama Rao, C.A., Vijay Kumar, P., Dixit, S. and Sikka, A.K., 2013. National Initiative on Climate Resilient Agriculture (NICRA), Research Highlights (2012-13). Central Research Institute for Dry land Agriculture, Hyderabad.

\section{How to cite this article:}

Manjunath, K.V., K. Shivaramu and Murthy, M.A. 2019. Knowledge and Adoption of Climate Resilient Agricultural Technologies by Paddy Growers. Int.J.Curr.Microbiol.App.Sci. 8(08): 1430-1442. doi: https://doi.org/10.20546/ijcmas.2019.808.167 